\title{
Erratum: Rubbing dynamics behavior of a flywheel shafting with a single point flexible support
}

\author{
Changliang Tang ${ }^{1}$, Dongjiang Han ${ }^{2}$, Jinfu Yang ${ }^{3}$ \\ Institute of Engineering Thermophysics, Chinese Academy of Sciences, Beijing, 100190, China \\ ${ }^{1}$ Corresponding author \\ E-mail: ${ }^{1}$ tangcl08@mails.tsinghua.edu.cn, ${ }^{2}$ handongjiang@iet.cn, ${ }^{3}$ yangjinfu@iet.cn
}

DOI https://doi.org/10.21595/jve.2017.19327

Check for updates

\section{Publisher's note regarding paper}

Changliang Tang, Dongjiang Han, Jinfu Yang Rubbing dynamics behavior of a flywheel shafting with a single point flexible support. Vol. 19, Issue 6, 2017, p. 4138-4154,

https://doi.org/10.21595/jve.2017.18480.

\section{The description of the correction}

The affiliation was misspelled in the paper originally submitted and finally approved (after the acceptance) by the Authors.

Incorrect affiliation:

Institute of Engineering Thermophysics, Chinese Academy of Sciences, Beijing, 100190, China

Correct affiliations:

${ }^{1}$ Key Laboratory of Modern Measurement and Control Technology, Ministry of Education, Beijing Information Science and Technology University, Beijing, 100192, China

${ }^{2,3}$ Institute of Engineering Thermophysics, Chinese Academy of Sciences,

Beijing, 100190, China 